\title{
Hirnblutung anlässlich einer oralen Antikoagulation - wer haftet?
}

\section{Andreas Wiget}

Dr. iur., Rechtsanwalt, Fachanwalt SAV Haftpflicht- und Versicherungsrecht, Mitglied der Medizinischen Kommission der Schweizerischen Ärzte-Krankenkasse

Das Bundesgericht wies die Klage eines Patienten ab, der Schadenersatz von seinem Hausarzt forderte, nachdem er anlässlich einer oralen Antikoagulation (OAK) eine Hirnblutung erlitten hatte und seither teilinvalid ist. Mit dem Vorwurf eines Kunstfehlers und unzureichender Aufklärung fand er vor Gericht kein Gehör. Im Folgenden werden einige Aspekte der Streitsache vom Rechtsvertreter des Hausarztes beleuchtet.

\section{Casum sentit dominus}

Der Grundsatz, wonach man einen erlittenen Schaden selber tragen müsse, es sei denn, ein anderer habe ihn zu verantworten, wird vom Bundesgericht hochgehalten. Der Arzt (oder die Ärztin) schuldet keinen Erfolg (Heilung usw.), sondern er hat einzig nach bestem Wissen auf den Erfolg hinzuarbeiten. Dessen Ausbleiben für sich allein führt zu keiner Haftung des Arztes, sofern er sorgfältig, lege artis, gehandelt hat. Im vorliegenden Fall ist dem Patienten der Nachweis eines Kunstfehlers bzw. einer Sorgfaltspflichtverletzung und ebenso die Verletzung einer Aufklärungspflicht misslungen.

Hémorragie cérébrale lors d'un traitement anticoagulant oral qui est responsable?

Le cas décrit dans le présent article démontre les limites de la responsabilité liée à l'activité médicale. Le médecin de famille dont il est question n'a pas été jugé responsable de l'hémorragie cérébrale de son patient sous traitement anticoagulant oral malgré l'atteinte à la santé permanente encourue par ce dernier. II s'agissait d'une part de pondérer les différents risques, dans le cas présent un risque élevé d'accident vasculaire cérébral sans traitement et un faible risque d'hémorragie cérébrale sous anticoagulants oraux. Dans un tel cas, on peut partir du principe que le patient consent au traitement. En revanche, il convient d'adapter le dosage du médicament (ici: Marcoumar) à la mesure régulière de I'INR et d'interrompre le traitement si nécessaire. Si la valeur-cible de I'INR est légèrement dépassée, les autres mesures thérapeutiques telles que l'administration de vitamine $\mathrm{K} 1$ sont écartées. Enfin, le cas décrit ici démontre la valeur probatoire d'une expertise selon qu'elle a été mandatée de manière unilatérale, commune ou par le tribunal.

\section{Behandlungsverlauf}

Der Patient litt seit Jahren über Vorhofflimmern und Herzarrhythmien und wurde vom Hausarzt in Zusammenarbeit mit Kardiologen des Kantonsspitals St.Gallen und des UniversitätsSpitals Zürich v.a. mit Cordarone und Marcoumar behandelt. Weil die bisherigen Therapien nicht anhaltend griffen, empfahl ein Zürcher Kardiologe im Sommer 2003 eine Lungenvenenisolation und verordnete die Abgabe von Cordarone $2 \times 400 \mathrm{mg}$ täglich und ebenso eine OAK. Mit dem Verabreichen von Marcoumar wurde noch zugewartet, weil beim Patienten eine Schulteroperation bevorstand. Nach deren Durchführung wurde das Cordarone auf $2 \times 200 \mathrm{mg}$ reduziert. Eine Woche später gab der Hausarzt dem Patienten wie vorgesehen Marcoumar mit einem INR-Zielwert von 2-3 ab, und zwar mit einer Anfangsdosis von $3 / 3 / 2$. Eine erste Kontrolle nach drei Tagen ergab einen INR von 2,3, worauf der Arzt die Dosis auf $1 / 2 / 3 / 4 / 3 / 4 / 1 / 2$ verminderte. Vier Tage später war der INR auf 3,3 gestiegen, worauf die Dosis erneut reduziert wurde ( $1 / 2$ täglich). Weitere fünf Tage erreichte der INR gar 4,5, worauf der Arzt das Marcoumar für zwei Tage absetzte und danach beabsichtigte, die Dosierung mit 1/4/1/4/1/2/1/4/1/4 fortzusetzen. Dazu kam es nicht mehr, weil der Patient im Zug eine Hirnblutung erlitten hatte. Seither ist er trotz Notoperation und weiteren Behandlungen teilinvalid. Beim Notfalleintritt betrug der INR 5,25.

\section{Medizinisches Privatgutachten}

Der vom Patienten beigezogene Anwalt schloss auf eine Verletzung der Sorgfalts- und der Aufklärungspflicht 
durch den Hausarzt. Beim damaligen Notoperateur, einem Neurochirurgen, holte er ein Gutachten ein. Nach dessen Ansicht war die Anfangsdosis zu hoch, nicht zuletzt aufgrund Erfahrungen des Hausarztes mit dem gleichen Patienten aus dem Vorjahr, als der INR bei umgekehrter Medikation - Cordarone wurde erst im Lauf einer OAK abgegeben - entgleist war (INR 5,2). Dem Arzt sei sodann vorzuwerfen, die Interaktion von Cordarone und Marcoumar nicht gekannt und beachtet zu haben. Weiter sei zu wenig eng kontrolliert und das Marcoumar zu spät abgesetzt worden, d.h. erst bei einem INR von 4,5 statt schon beim Überschreiten des Zielwerts mit 3,3. Zudem hätte der Arzt nach gutachterlicher Ansicht dem Patienten Vitamin K1 geben müssen.

\section{FMH-Begutachtung}

Die Haftpflichtversicherung des Arztes lehnte das hinter ihrem Rücken eingeholte Gutachten ab. Man einigte sich aber auf eine neutrale Begutachtung. Die von der FMH-Gutachterstelle eingesetzten Gutachter, ein Allgemeinmediziner und ein Neurologe, stellten fest, dass 2003 einheitliche Richtlinien zur OAK nicht bestanden. Sie kamen aufgrund des Studiums der Krankengeschichte und gestützt auf ausländische Richtlinien und Studien zum Schluss, die OAK sei indiziert und das Vorgehen des Hausarztes bezüglich Dosierung, Kontrolle und Stopp der Abgabe von Marcoumar korrekt gewesen. Sie billigten ihm aufgrund des regen Schrift-

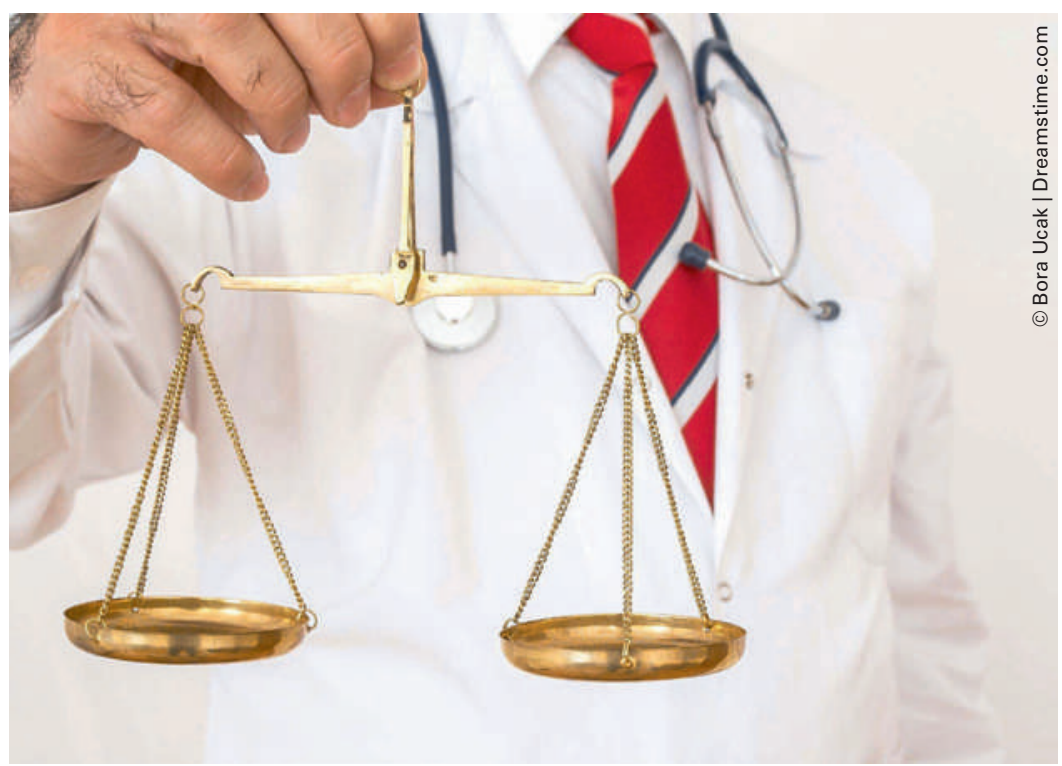

Welcher Wissensstand ist für die Beurteilung, ob dem Hausarzt ein Kunstfehler unterlaufen ist, massgeblich? Der eines Hausarztes oder der eines Spezialisten? Und war der Wissensstand in dem Jahr entscheidend, in dem die Behandlung stattfand, oder zählt die heutige Sicht? verkehrs mit den Kardiologen zu, die Abklärungen und Therapien sorgfältig geplant und durchgeführt zu haben. Der vom Neurochirurgen erhobene Vorwurf, der Hausarzt hätte die Interaktion von Marcoumar mit Cordarone nicht gekannt oder bei der Dosierung nicht berücksichtigt, gehe fehl. Die Gutachter wiesen auf eine

\section{Die Gutachter wiesen auf eine Vielzahl von}

Medikamenten, Alkoholkonsum und die Ernährung hin, die die Wirkung der OAK beeinflussten.

Vielzahl von Medikamenten, Alkoholkonsum und die Ernährung hin, die die Wirkung der OAK beeinflussten. «Dies ist mit ein Grund, warum die Wirkung der Antikoagulantien durch regelmässige Kontrollen des INR festgestellt werden muss». Die Blutung sei zwar auf die OAK zurückzuführen, müsse aber «als Restrisiko der Behandlung betrachtet werden. Hätte man andererseits die orale Antikoagulation nicht durchgeführt, wäre der Patient einem erheblichen Risiko für einen zerebralischämischen Infarkt ausgesetzt gewesen bzw. hätte der geplante Eingriff der Pulmonalvenenisolation nicht durchgeführt werden können».

\section{Rechtliche Begutachtung}

Der Patient liess darauf ein Rechtsgutachten einer medizinisch-juristischen Gutachterstelle erstellen. Nach Ansicht der Gutachter hatte der Arzt ihn unzureichend über die synergetische Wirkung von Cordarone und Marcoumar aufgeklärt. Ebenso hätte er ihm die Risiken bei einem INR von 4,5 erläutern und ihn zur vorübergehenden Reduktion oder gar zur Einstellung der Erwerbstätigkeit anhalten und ihm empfehlen müssen, sich ständig von einer Drittperson begleiten zu lassen. Weder der Arzt noch die Haftpflichtversicherung konnten diese Beurteilung nachvollziehen.

\section{Klage vor Gericht und gerichtliche Begutachtung}

Im Mai 2009 reichte der Patient beim Kreisgericht Rorschach Klage ein: Der Arzt sei zu verpflichten, ihm für den erlittenen Erwerbsausfall sowie Haushalts- und Betreuungsschaden Schadenersatz und als Genugtuung 1,6 Mio. Franken zu bezahlen. Das Gericht wies die Klage gestützt auf das FMH-Gutachten und verschiedene ihm zur Kenntnis gebrachte Studien zu Risiken der OAK im Februar 2012 ab, worauf der Patient ans Kantonsgericht St. Gallen gelangte. Dieses beauftragte einen Spezialarzt für Innere Medizin und Infektiologie, ein gerichtliches Gutachten zu erstatten. Wie schon die Vorgut- 
achter wies er zwar auf das mit einer OAK verbundene Risiko einer Blutung hin, aber ebenso auf das ungleich höhere Risiko eines Hirninfarkts beim Verzicht auf die OAK; auch er hielt diese angesichts der Umstände für indiziert. Aufgrund der 2002 gemachten Erfahrungen seien die hohe Anfangsdosis und die Erhaltungsdosen auch bei gleichzeitiger Medikation mit Cordarone korrekt gewesen, ebenso die Reduktion des Marcoumars bei einem INR von 3,3 und das Absetzen bei einem solchen von 4,5. Ebenso lehnte er die geforderten zusätzlichen

\section{Als Arzt lässt sich praktizieren, ohne mit einem Bein im Gefängnis zu stehen.}

Vorsichtsmassnahmen wie Abgabe von Vitamin K1, Reduktion der Arbeitstätigkeit usw. ab. Der Gutachter meinte zwar, ein Hausarzt müsse die Interaktionen von häufig verwendeten Medikamenten kennen, aber Cordarone sei kein von Hausärzten häufig verschriebenes Medikament. Im vorliegenden Fall habe der Hausarzt sodann auch in Unkenntnis dieser Interaktion korrekt gehandelt, indem er den INR ausreichend kontrolliert und die richtigen Schlüsse daraus gezogen habe. Am 26. Mai 2015 wies das Kantonsgericht die Berufung des Patienten ab, worauf dieser Beschwerde beim Bundesgericht erhob. Der Beschwerde war kein Erfolg beschieden (siehe das Urteil 4A_343/2015 vom 21. Oktober 2015, zu finden unter www.bger.ch $\rightarrow$ Rechtsprechung $\rightarrow$ Rechtsprechung gratis $\rightarrow$ weitere Urteile ab 2000 $\rightarrow$ 4A 343/2015 im Suchfeld eingeben). Die finanziellen Konsequenzen für den (nicht rechtsschutzversicherten) Patienten sind verheerend: Für Gerichts-, Expertise- und Gegenanwaltskosten muss er gegen 250000 Franken bezahlen. Die Kosten für seinen Anwalt und die Privatexpertisen werden sich auf weitere mindestens 100000 Franken belaufen.

\section{Folgerungen}

\section{Richtige Behandlung}

Im Gegensatz zum eingangs erwähnten römisch-rechtlichen Grundsatz können es Juristen, nicht nur Anwälte, sondern auch Richter, oft kaum glauben, dass kein Haftpflichtiger vorhanden sein soll. «Hätte er nicht doch ...? Wäre es nicht doch ...?», frägt man sich jeweils. Ganz unverständlich war dies im vorliegenden Fall nicht, denn der medizinische Laie musste einiges zur Kenntnis nehmen, womit er nicht gerechnet hätte. Erwähnt sei die oben beschriebene Interaktion von Cordarone mit Marcoumar, die trotz ihrer Auswirkungen auf den INR keine entscheidende Bedeutung hatte, weitere hier interessierende Wechselwirkungen (z.B. mit Voltaren), die unterschiedlichen Halbwertszeiten von blutstillenden Mitteln, die Frage der Wirkung von Vitamin K1 usw. Als Jurist erwartet man, dass der behandelnde Arzt aufgrund solcher Umstände besondere Vorkehren zu treffen habe, und man wundert sich, weshalb auch die im Fall involvierten Kardiologen insbesondere die Interaktion mit Cordarone nie auch nur mit einem Wort erwähnten. Man wurde von den Gutachtern aber eines Besseren belehrt: Marcoumar interagiert mit verschiedensten Medikamenten, Alkohol und etlichen Nahrungsmitteln. Jeder Patient reagiert wieder anders, und es kommt die Unzuverlässigkeit der Patienten bei der Medikamenteneinnahme dazu. Die Entwicklung des INR lässt sich aus diesen Gründen nicht voraussehen. Was einzig zählt, sind regelmässige Kontrollen und die richtige Reaktion des Arztes, wenn der INR-Zielwert über- oder unterschritten wird (Anpassen der Dosierung, evtl. Absetzen des Medikaments). Die lapidare Feststellung im FMH-Gutachten, wonach der behandelnde Arzt einzig die Wirkung der Antikoagulantien durch regelmässige Kontrollen des INR festzustellen habe, ansonsten aber bei einem "Nicht-High-risk»-Patienten wie im vorliegenden Fall nichts Besonderes vorkehren müsse, ist in ihrer Einfachheit für einen Juristen schwer verständlich und war unter anderem ein Grund, weshalb das Kantonsgericht zusätzlich ein gerichtliches Gutachten anordnete.

Massgebend sind also nicht heutige Erkenntnisse und ebensowenig die Kenntnisse eines Spezialisten.

Nunmehr ist jedoch die gutachterlich vertretene Ansicht, wonach eine regelmässige Kontrolle des INR genüge, solange es sich nicht um einen Risikopatienten handle, höchstrichterlich abgesegnet. Als sehr fraglich wird aufgrund der Ergebnisse von Studien die Abgabe von Vitamin K1 bezeichnet. Und ist der Arzt insbesondere bei einem erfahrenen Patienten nicht gehalten, ihn jedesmal auf irgendwelche Gefahren aufmerksam zu machen; die Abgabe des Antikoagulations-Ausweises ist ausreichend.

\section{Keine ausufernde Haftung für Ärzte}

Generell scheint die Angst, dass «amerikanische Verhältnisse» Einzug in die schweizerische Rechtsprechung fänden, unberechtigt. Als Arzt lässt sich praktizieren, ohne mit einem Bein im Gefängnis zu stehen. Gerade kürzlich fällte das Bundesgericht das wegweisende Urteil 4A_137/2015 vom 19. August 2015, wo es darum ging, dass ein Gynäkologe einen Dammriss nach Geburt zwar genäht, aber nicht erkannt hatte, dass ein (okkulter) Dammriss III ${ }^{\circ}$ eingetreten war und damit eine Verlet- 
zung des äusseren analen Sphinkters, was zu einer Stuhlinkontinenz führte. Eine von ihm vorgenommene Rektaluntersuchung hatte der Arzt nicht dokumentiert. Das Bundesgericht folgte seiner Argumentation, wonach aus dem Fehlen einer Dokumentation nicht auf die Nichtvornahme der Untersuchung geschlossen werden dürfe, denn bei der Rektaluntersuchung handle es sich um eine routinemässige Kontrolle, die nicht dokumentiert werden müsse. Eine Haftung des Arztes wurde daher abgelehnt.

\section{Massgeblicher Wissensstand}

Entscheidend für die Gutachter wie auch für die Gerichte war im OAK-Fall explizit der «Wissensstand eines Hausarztes (im Jahr) 2003», dem Jahr, als die Behandlung stattgefunden hatte. Massgebend sind also nicht heutige Erkenntnisse und ebensowenig die Kenntnisse eines Spezialisten. Die FMH-Gutachterstelle wählte aus diesem Grund bewusst einen Allgemeinmediziner als Hauptgutachter aus, und im gleichen Sinne wies das Kantonsgericht den Gerichtsgutachter an. Notabene waren für das Bundesgericht auch im Dammrissfall die konkreten Umstände massgebend, und zwar prüfte es die Dokumentationspflicht aus der Sicht eines Arztes im Jahr 1993, als der Damm gerissen war, und nicht aus heutiger Sicht. Ob eine Sorgfaltspflichtverletzung vorliegt oder nicht, ist stets ex-ante und nicht ex-post zu ermitteln.

\section{Beweiswert von Gutachten}

Vom Patienten einseitig eingeholte Gutachten, sog. Privatgutachten, gelten zivilprozessual als Parteibehauptungen ohne Beweiswert (so das Bundesgericht in einem neuen Urteil 4A_178/2015 Erw. 2.6). In der Praxis kann ein Parteigutachten allerdings durchaus etwas bewirken, nämlich wenn es dazu dient, Fehler eines neutralen Gutachtens aufzuzeigen und dessen Glaubwürdigkeit zu erschüttern, und damit den Richter dazu bringt, eine Ergänzung des Gutachtens oder gar eine
Oberexpertise in Auftrag zu geben. Diese Wirkung konnte im vorliegenden Fall das neurochirurgische Gutachten von vornherein nicht haben, weil es erstellt wurde, bevor das FMH-Gutachten vorlag. Vom Kantonsgericht wurde ihm aber auch deshalb kein Beweiswert zugemessen, weil dem Gutachter nicht die ganze Krankengeschichte vorlag, seine Arbeit folglich nicht vollständig war, und zudem nicht klar wurde, worauf er seine wertenden Äusserungen zum Vorgehen des betroffenen Hausarztes stützte und ob er diese aus der Sicht eines Fach- oder Hausarztes machte.

\section{Vom Patienten einseitig eingeholte Gutachten gelten zivilprozessual als Parteibehauptungen ohne Beweiswert.}

Anders verhält es sich bei FMH-Gutachten, die von den Beteiligten gemeinsam in Auftrag gegeben werden und denen ein ähnlich hoher Beweiswert wie einem gerichtlichen Gutachten zukommt, sofern es die Anforderungen erfüllt, die das Bundesgericht an eine Expertise stellt. Generell hat ein Gutachten vollständig, nachvollziehbar und schlüssig zu sein, um vollen Beweis zu erbringen. Es ist für ein solches entscheidend, ob es für die streitigen Belange umfassend ist, auf allseitigen Untersuchungen beruht, die geklagten Beschwerden berücksichtigt, in Kenntnis der Vorakten (Anamnese) abgegeben worden ist und in der Beurteilung der medizinischen Zusammenhänge und in der Beurteilung der medizinischen Situation einleuchtet (Urteil des Bundesgerichts 4A_14/2011 Erw. 2.2).

Erfüllt ein Gutachten diese Anforderungen nicht, ist es nutzlos, und man kann sich die Kosten dafür sparen. Dasselbe muss auch in vielen Fällen von Rechtsgutachten gesagt werden, da darin rechtliche Fragen aufgeworfen werden, «deren Beurteilung» - so das Kantonsgericht - «in diesem Prozess einzig dem Gericht obliegt». Das Gericht zieht den apodiktischen Schluss: «Vorliegend ist dieses Gutachten daher unbeachtlich.» 